\title{
Modelling the Effects of Snail Control and Health Education in Clonorchiasis Infection in Foshan, China
}

\author{
Shujing Gao $\mathbb{D}^{1},{ }^{1}$ Ruixia Yuan, ${ }^{2}$ Yujiang Liu, ${ }^{1}$ and Xinzhu Meng $\mathbb{D}^{3}$ \\ ${ }^{1}$ Key Laboratory of Jiangxi Province for Numerical Simulation and Emulation Techniques, Gannan Normal University, \\ Ganzhou 341000, China \\ ${ }^{2}$ College of Business Economics, Shanghai Business School, Shanghai 200336, China \\ ${ }^{3}$ College of Mathematics and Systems Science, Shandong University of Science and Technology, Qingdao 266590, China \\ Correspondence should be addressed to Shujing Gao; gaosjmath@126.com
}

Received 18 January 2019; Revised 11 May 2019; Accepted 16 June 2019; Published 9 July 2019

Academic Editor: Hassan Zargarzadeh

Copyright (C) 2019 Shujing Gao et al. This is an open access article distributed under the Creative Commons Attribution License, which permits unrestricted use, distribution, and reproduction in any medium, provided the original work is properly cited.

Clonorchiasis is the most important food-borne parasitic disease in China. In this paper, a mathematical model of clonorchiasis sinensis is proposed to mimic its transmission dynamics to assess the effects of intervention strategies such as snail control, health education, and chemotherapy. A threshold dynamics in terms of the basic reproductive number $R_{0}$ has been established; that is, if $R_{0}<1$, the disease dies out and the disease-free periodic solution is globally asymptotically stable, and if $R_{0}>1$, then the disease breaks out. The effects of different control measures are compared by numerical simulations. The numerical results suggest that it is necessary to strengthen health education and improve faeces management and illustrate that snail control is the most effective way to be implemented in the clonorchiasis sinensis control in Foshan.

\section{Introduction}

Clonorchiasis sinensis (C. sinensis) is a major food-borne parasitosis, which is actively infected in China, the Democratic People's Republic of Korea, the Republic of Korea, Russia, and Viet Nam [1]. Currently, it is estimated that more than 200 million people are at risk of infection [2], and about 35 million people are infected globally, including 15 million in China [3]. There are two major endemic regions in China-namely, provinces in the southeast, including Guangdong and Guangxi, and provinces in the northeast, such as Heilongjiang and Jilin [4]. Human beings are infected through ingestion of raw or undercooked fish which contains the metacercariae of liver flukes [5-7]. The clonorchiasis infection (CI) may cause serious liver and biliary system damage and affect many sectors of human health $[4,8]$.

Foshan is located in the central of Pearl River Delta in Guangdong. It enjoys subtropical monsoon climate, with warm weather, abundant sunshine, and rainfall. Agriculture is based on rice cultivation and freshwater aquaculture industry. Mulberry fish pond is above $50 \%$ of the total farmland area. Local residents like to eat raw fish. Additionally, coupled with misconceptions, such as the belief that consumption of alcohol or spicy food can prevent infection [9], Foshan has been becoming a heavily clonorchiasis-endemic area. Therefore, in order to reduce the CI rate, a number of strategies have been proposed including chemotherapy (morbidity control with praziquantel), information, education, communication, and faeces management (sanitation improvement and animal management). In [10], it is reported that after the implement of integrated control strategies, the CI rate of population in Foshan decreased dramatically from $56.84 \%$ in 1989 to $3.47 \%$ in 2000 . But the rate shows the resurgence since 2005 owing to the general investigation project eased from 2001 to 2004 and the tradition of consuming raw freshwater fish or shellfish resuscitated (see Figure 1). This shows that health education does play important role in the control of C. sinensis.

The amphibious snail is one of intermediate hosts of $C$. sinensis. As far as we know, snail control with molluscicide is one of important control measures for schistosomiasis but has not been widely applied in practice for clonorchiasis control. However, some biologists have tried to study the role of snail-killing in controlling the spread of C. sinensis. For example, Yang [11] carried out comprehensive measures 


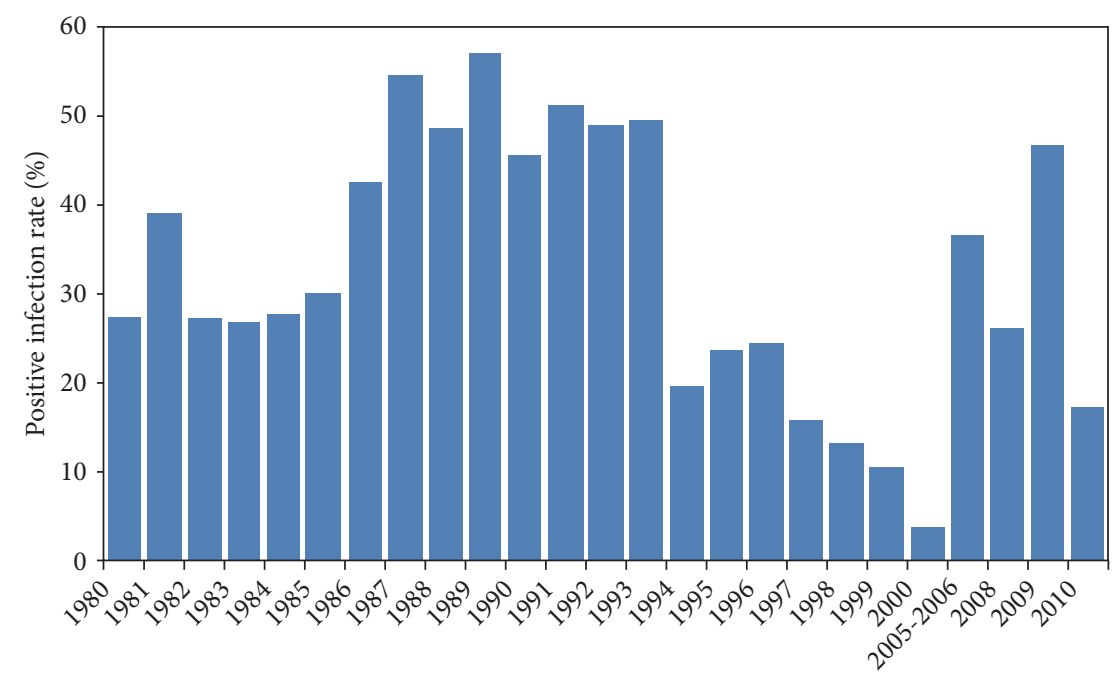

Year

FIGURE 1: Positive infection rate in Foshan from 1980 to 2010.

in Daliang Village, Yangshan County, Guangdong Province, including health education, chemotherapy, and snail control with molluscicide. The CI rate decreased from $48.2 \%$ in 1975 to $2.9 \%$ in 1976 to 0 in $1979-1982$. Therefore, it is of great practical significance to assess different tools and strategies for large-scale control of clonorchiasis by using mathematical modelling.

There have been studies of modeling clonorchiasis associated with the human being in China $[12,13]$. However, none of the studies were to quantitatively consider the relationship between CI rate and the number of molluscicide to spray when chemotherapy and health education are implemented in combination. Mathematical models can be important for determining the optimal number of molluscicide spraying so as to control the transmission of clonorchiasis. In this paper, a clonorchiasis spread model with an integrated strategy is developed and studied, in which seasonal variation and impulsive control are considered.

This paper is organized as follows. In Section 2, we propose a $C$. sinensis model with pulse snail-killing, health education, and chemotherapy control in periodic environment. Some results are stated which will be essential to our proof. We calculate the basic reproductive number for the model in Section 3. In Section 4, we illustrate that the basic reproductive number serves as a threshold parameter that determines the disease to be extinction or endemic. In Section 5, different control programs are compared and sensitivity analysis is done to evaluate the snails control strategy by numerical simulations. Section 6 gives a brief discussion and future work.

\section{Model and Preliminaries}

Clonorchiasis belongs to vector-borne disease including two intermediate hosts: snail and fish. C. sinensis is transmitted indirectly among the human hosts, first intermediate snails, and second intermediate fishes in the sense that freeswimming stages (miracidia, cercariae) and ingestion stage (metacercariae) are interposed. In addition to human beings, specially, many mammals, such as dogs, cats, pigs, rodents, foxes, and possibly any fish eating mammal, can serve as definitive hosts for $C$. sinensis and humans are the main definitive hosts [14]. In order to simplify the mathematical model, we only consider human hosts here. Figure 2 shows a schematic description of the transmission of clonorchis sinensis in definitive host, snail host, and fish host.

In this section, we mainly formulate an impulsive epidemic model to describe the transmission dynamics of $C$. sinensis.

We firstly formulate a model for the spread of $C$. sinensis incorporating health education and chemotherapy strategies. The total human population at any time $t$, denoted by $N(t)$, is the sum of individual populations in each compartment which includes susceptible $S_{h}(t)$, infected $I_{h}(t)$, and recovered $R_{h}(t)$. We assume that the total human population remains a constant, denoting $N_{h}(t)=N_{h}$ for all $t \geq 0$.

Following the idea of Dai and Gao [13], we divide the snail population and fish population into disjoint classes: susceptible $\left(S_{s}, S_{f}\right)$ and infected $\left(I_{s}, I_{f}\right)$, respectively. We suppose that the infection rates of susceptible human, susceptible snail, and susceptible fish are described by

$$
\begin{aligned}
& \beta_{1}(t) S_{h}(t) I_{f}(t), \\
& \beta_{2}(t) S_{s}(t) I_{h}(t), \\
& \beta_{3}(t) S_{f}(t) I_{s}(t),
\end{aligned}
$$

respectively, where $\beta_{1}(t)$ is per capita (successful) infection rate of human hosts by one fish at time $t, \beta_{2}(t)$ is per capita (successful) infection rate of snails by one human at time $t$, and $\beta_{3}(t)$ is per capita (successful) infection rate of fish by one snail at time $t$. 


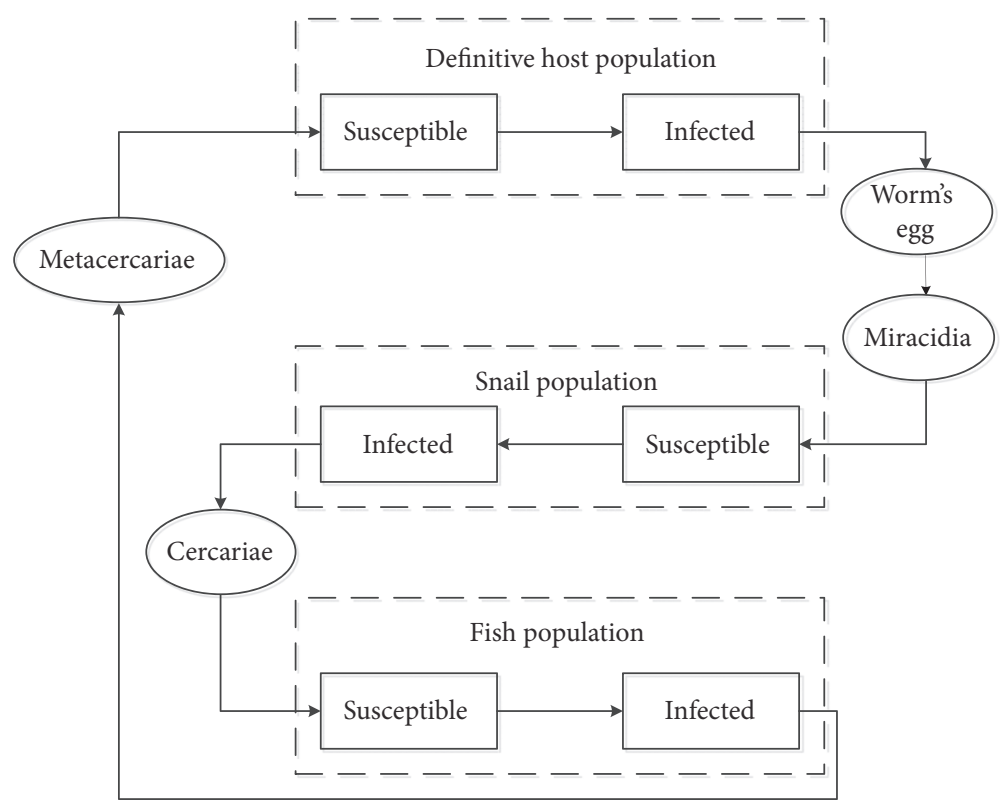

FIgURE 2: A transmission diagram of Clonorchis sinensis.

Some residents would temporarily change their bad eating habit owing to health education. Let $\alpha(t)$ denote the proportion of human host at time $t$ from susceptible to recovered human hosts owing to health education, $\gamma(t)$ is the recovery rate due to chemotherapy, and $\delta(t)$ is the proportion of human host at time $t$ of transition from recovered to susceptible human hosts due to the loss of heath awareness and misconceptions. Suppose that sail population and fish population do not result in death and increase at periodic recruitment rates $\Lambda_{2}(t)$ and $\Lambda_{3}(t)$ and also decrease at the natural death rates $\mu_{2}(t)$ and $\mu_{3}(t)$, respectively. Incorporating above assumptions, we have the following model, in which seasonal variation and control measures (health education and chemotherapy) are taken into consideration:

$$
\begin{aligned}
S_{h}^{\prime}(t)= & \mu_{1}(t) N_{h}(t)-\beta_{1}(t) S_{h}(t) I_{f}(t)-\alpha(t) S_{h}(t) \\
& -\mu_{1}(t) S_{h}(t)+\delta(t) R_{h}(t), \\
I_{h}^{\prime}(t)= & \beta_{1}(t) S_{h}(t) I_{f}(t)-\gamma(t) I_{h}(t)-\mu_{1}(t) I_{h}(t), \\
R_{h}^{\prime}(t)= & \alpha(t) S_{h}(t)+\gamma(t) I_{h}(t)-\mu_{1}(t) R_{h}(t) \\
& -\delta(t) R_{h}(t), \\
S_{s}^{\prime}(t)= & \Lambda_{2}(t)-\beta_{2}(t) S_{s}(t) I_{h}(t)-\mu_{2}(t) S_{s}(t), \\
I_{s}^{\prime}(t)= & \beta_{2}(t) S_{s}(t) I_{h}(t)-\mu_{2}(t) I_{s}(t), \\
S_{f}^{\prime}(t)= & \Lambda_{3}(t)-\beta_{3}(t) S_{f}(t) I_{s}(t)-\mu_{3}(t) S_{f}(t), \\
I_{f}^{\prime}(t)= & \beta_{3}(t) S_{f}(t) I_{s}(t)-\mu_{3}(t) I_{f}(t),
\end{aligned}
$$

where all parameters are positive, periodic, andcontinuous functions with period 12 (months).
Since $R_{h}(t)=N_{h}-S_{h}(t)-I_{h}(t)$, then (2) can yield

$$
\begin{aligned}
S_{h}^{\prime}(t)= & \left(\mu_{1}(t)+\delta(t)\right) N_{h}-\beta_{1}(t) S_{h}(t) I_{f}(t) \\
& -\left(\alpha(t)+\mu_{1}(t)+\delta(t)\right) S_{h}(t) \\
& -\delta(t) I_{h}(t), \\
I_{h}^{\prime}(t)= & \beta_{1}(t) S_{h}(t) I_{f}(t)-\gamma(t) I_{h}(t)-\mu_{1}(t) I_{h}(t), \\
S_{s}^{\prime}(t)= & \Lambda_{2}(t)-\beta_{2}(t) S_{s}(t) I_{h}(t)-\mu_{2}(t) S_{s}(t), \\
I_{s}^{\prime}(t)= & \beta_{2}(t) S_{s}(t) I_{h}(t)-\mu_{2}(t) I_{s}(t), \\
S_{f}^{\prime}(t)= & \Lambda_{3}(t)-\beta_{3}(t) S_{f}(t) I_{s}(t)-\mu_{3}(t) S_{f}(t), \\
I_{f}^{\prime}(t)= & \beta_{3}(t) S_{f}(t) I_{s}(t)-\mu_{3}(t) I_{f}(t) .
\end{aligned}
$$

Snail control is conducted with molluscicide in fish ponds and canals. Note that molluscicides are most commonly sprayed to be taken on different dose and different timeinterval in a year. The phenomenon exhibits impulsive effects on the transmission of $C$. sinensis. Suppose the number of molluscicide to spray is $q$ in one year, $t_{k}(k \in \mathbb{N})$ is the pulse time, and the elimination rate of snails at time $t_{k}$ is $\theta_{k}$. Then we have impulsive equations:

$$
\begin{aligned}
& S_{s}\left(t^{+}\right)=\left(1-\theta_{k}\right) S_{s}(t), \\
& I_{s}\left(t^{+}\right)=\left(1-\theta_{k}\right) I_{s}(t),
\end{aligned}
$$

for $t=t_{k}$,

where $S_{s}\left(t^{+}\right)=\lim _{h \longrightarrow 0^{+}} S_{s}(t+h), I_{s}\left(t^{+}\right)=\lim _{h \longrightarrow 0^{+}} I_{s}(t+h)$, and $\theta_{k+q}=\theta_{k}, t_{k+q}=t_{k}+12(k \in \mathbb{N})$.

The system consisting of (3) and (4) is an impulsive differential system. For simplicity, we will refer to the system as "the model system" in the rest of this paper. 
Based on the biological background of the model system, we always assume that all solutions of the model system satisfy the following initial conditions:

$$
S_{i}\left(0^{+}\right) \geq 0, \quad \text { and } I_{i}\left(0^{+}\right) \geq 0 \text { for } i=h, s, f .
$$

It is not difficult to prove that the positive cone of $R_{+}^{6}$ is flow invariant relative to the model system.

Let $N_{s}(t)$ and $N_{f}(t)$ denote the density of sail population and fish population at time $t$, respectively. From (3) and (4), we have

$$
\begin{aligned}
N_{\mathrm{s}}^{\prime}(t) & =\Lambda_{2}(t)-\mu_{2}(t) N_{s}(t), \quad t \neq t_{k}, k \in \mathbb{N}, \\
N_{s}\left(t^{+}\right) & =\left(1-\theta_{k}\right) N_{s}(t), \quad t=t_{k}, k \in \mathbb{N},
\end{aligned}
$$

and

$$
N_{f}^{\prime}(t)=\Lambda_{3}(t)-\mu_{3}(t) N_{f}(t)
$$

For a continuous, positive $\omega$-periodic function $f(t)$, we set $f^{M}=\sup _{t \in[0, \omega]} f(t)$ and $f^{L}=\inf _{t \in[0, \omega]} f(t)$. Define a set

$$
\begin{aligned}
\Omega & =\left\{\left(S_{h}(t), I_{h}(t), S_{s}(t), I_{s}(t), S_{f}(t), I_{f}(t)\right)\right. \\
& \in R_{+}^{6} \mid 0 \leq S_{h}(t)+I_{h}(t) \leq N_{h}, 0 \leq S_{s}(t)+I_{s}(t) \\
& \left.\leq N_{s}^{*}, 0 \leq S_{f}(t)+I_{f}(t) \leq N_{f}^{*}\right\},
\end{aligned}
$$

where $N_{s}^{*}=\Lambda_{2}^{M} / \mu_{2}^{L}$ and $N_{f}^{*}=\Lambda_{3}^{M} / \mu_{3}^{L}$.

Lemma 1. $\Omega$ is a positively invariant set of the model system.

Proof. Let $\left(S_{h}(t), I_{h}(t), S_{s}(t), I_{s}(t), S_{f}(t), I_{f}(t)\right)$ be any positive solution of the model system with initial condition (5).

For system (6), we consider the following auxiliary system:

$$
\begin{aligned}
H_{s}^{\prime}(t) & =\Lambda_{2}(t)-\mu_{2}(t) H_{s}(t), \\
H_{s}\left(0^{+}\right) & =N_{s}\left(0^{+}\right) .
\end{aligned}
$$

Thus we have

$$
H_{s}^{\prime}(t) \leq \Lambda_{2}^{M}-\mu_{2}^{L} H_{s}(t)<0, \quad \text { if } H_{s}(t)>\frac{\Lambda_{2}^{M}}{\mu_{2}^{L}}=N_{s}^{*} .
$$

Then it follows from comparison theorem, we have $\limsup \sup _{t \rightarrow+\infty} N_{s}(t) \leq N_{s}^{*}$. Applying similar method, we can obtain that $\lim \sup _{t \rightarrow+\infty} N_{f}(t) \leq N_{f}^{*}$. This completes the proof.

Lemma 2. Consider the following impulsive differential equation:

$$
\begin{aligned}
& z^{\prime}(t)=a(t)-b(t) z(t), \quad t \neq t_{k}, \\
& z\left(t^{+}\right)=\left(1-\theta_{k}\right) z(t), \quad t=t_{k},
\end{aligned}
$$

where $a(t)$ and $b(t)$ are continuous and positive $\omega$-periodic functions, and there is a positive integer q such that $\theta_{k+q}=\theta_{k}$ and $t_{k+q}=t_{k}+\omega$ for all $k \in N$. Then there exists a unique positive periodic solution of system (11)

$$
\begin{aligned}
z^{*}(t)= & \prod_{l=1}^{i}\left(1-\theta_{l}\right) z_{0}^{*} e^{\int_{n \omega+t_{0}}^{t}-b(\tau) d \tau} \\
+ & e^{\int_{n \omega+t_{i}}^{t}-b(\tau) d \tau} \int_{n \omega+t_{i}}^{t} a(s) e^{\int_{n \omega+t_{i}}^{s} b(\tau) d \tau} d s \\
+ & \sum_{l=1}^{i} \prod_{j=1}^{i}\left(1-\theta_{j}\right) e^{\int_{n \omega+t_{j-1}}^{t}-b(\tau) d \tau} \\
& \cdot \int_{n \omega+t_{j-1}}^{n \omega+t_{j}} a(s) e^{\int_{n \omega+t_{j-1}}^{s} b(\tau) d \tau} d s,
\end{aligned}
$$

for $n \omega+t_{i}<t \leq n \omega+t_{i+1}, \quad i=0,1, \ldots, q-1$, which is globally asymptotically stable, where

$$
\begin{aligned}
z_{0}^{*}= & {\left[1-\prod_{l=1}^{q}\left(1-\theta_{l}\right) e^{\int_{n \omega+t_{0}}^{n \omega+t_{q}}-b(\tau) d \tau}\right]^{-1} } \\
& \cdot \sum_{l=1}^{q} \prod_{j=l}^{q}\left(1-\theta_{j}\right) e^{\int_{n \omega+t_{j-1}}^{n \omega+t_{q}}-b(\tau) d \tau} \\
& \cdot \int_{n \omega+t_{j-1}}^{n \omega+t_{j}} a(s) e^{\int_{n \omega+t_{j-1}}^{s} b(\tau) d \tau} d s .
\end{aligned}
$$

The proof of Lemma 2 can be seen in the Appendix.

By Lemma 2, it is easy to see that the following conclusion holds true.

Theorem 3. The model system always has a unique diseasefree periodic solution $\left(S_{h}^{*}(t), 0, S_{s}^{*}(t), 0, S_{f}^{*}(t), 0\right)$.

Before proving the main results, we introduce the following notations.

Let $\left(R^{n}, R_{+}^{n}\right)$ be the standard ordered $\mathrm{n}$-dimensional Euclidean space with a norm $\|\cdot\|$. For $\varrho, v \in R^{n}$, we denote $\varrho \geq v$ if $\varrho-v \in R_{+}^{n} ; \varrho>v$ if $\mu-\nu \in R_{+}^{n} \backslash\{0\}$; and $\varrho \gg v$ if $\varrho-\nu \in \operatorname{Int}\left(R_{+}^{n}\right)$, respectively.

Let $\Psi_{A}(t, s)(t \geq s)$ be the evolution operator of the linear $\omega$-periodic system

$$
\dot{x}(t)=A(t) x(t), \quad x \in \mathbb{R}^{n}
$$

Consider the linear impulsive system

$$
\begin{aligned}
& x^{\prime}(t)=A(t) x, \quad t \neq t_{k}, k \in \mathbb{N}, \\
& x\left(t^{+}\right)=P_{k} x\left(t_{k}\right), \quad t=t_{k}, k \in \mathbb{N}, \\
& x\left(0^{+}\right)=x(0)=x_{0}, \quad t_{0}=0,
\end{aligned}
$$

which satisfies the following three conditions:

$\left(\mathrm{a}_{1}\right) A(\cdot) \in \mathrm{C}\left(\mathbb{R}, \mathbb{R}^{n \times n}\right), A(t+\omega)=A(t)$, where $\omega$ is a positive real number. 
$\left(\mathrm{a}_{2}\right) P_{k} \in \mathbb{R}^{n \times n}$, det $P_{k} \neq 0, t_{k}<t_{k+1}(k \in \mathbb{N})$, and $\lim _{k \rightarrow+\infty} t_{k}=+\infty$.

$\left(\mathrm{a}_{3}\right)$ There exists $q \in \mathbb{N}_{+}$such that $P_{k+q}=P_{k}, t_{k+q}=t_{k}+\omega$ $(k \in \mathbb{N})$.

Denote

$$
\Phi_{A P_{k}}(\omega):=\prod_{i=1}^{q}\left(P_{q-i+1} \Psi_{A}\left(t_{q-i+1}, t_{q-i}\right)\right) .
$$

By the Perron-Frobenius theorem, its spectral radius $r\left(\Phi_{A P_{k}}(\omega)\right)$ is the principal eigenvalue of $\Phi_{A P_{k}}(\omega)$ in the sense that it is simple and admits an eigenvector $v^{*} \gg 0$.

The following lemma is useful for our future discussion.
Lemma 4 (see [15]). Let $\eta=(1 / \omega) \ln r\left(\Phi_{A P_{k}}(\omega)\right)$. Then there exists a positive $\omega$-periodic function $v(t)$ such that $e^{\eta t} v(t)$ is a solution of system (15).

\section{The Basic Reproductive Number $R_{0}$}

To use the computation approach of the basic reproductive number (see [15]), we set $x=\left(x_{1}, x_{2}, x_{3}, x_{4}, x_{5}, x_{6}\right)^{T}=$ $\left(I_{h}, I_{s}, I_{f}, S_{h}, S_{s}, S_{f}\right)^{T}$ and rewrite system (3) as

$$
x^{\prime}(t)=\mathscr{F}(t, x(t))-\mathscr{V}(t, x(t)),
$$

where $\mathscr{F}$ is the new infection rate and $\mathscr{V}$ is the decay rate or transfer rate, and

$$
\mathscr{F}(t, x)=\left(\begin{array}{c}
\beta_{1}(t) x_{4}(t) x_{3}(t) \\
\beta_{2}(t) x_{5}(t) x_{1}(t) \\
\beta_{3}(t) x_{6}(t) x_{2}(t) \\
0 \\
0 \\
0
\end{array}\right),\left(\begin{array}{c}
\left.\mu_{1}(t)+\gamma(t)\right) x_{1}(t) \\
\mu_{2}(t) x_{2}(t) \\
\mu_{3}(t) x_{3}(t) \\
-\left(\mu_{1}(t)+\delta(t)\right) N_{h}+\beta_{1}(t) x_{4}(t) x_{3}(t)+\left(\alpha(t)+\mu_{1}(t)+\delta(t)\right) x_{4}(t)+\delta(t) x_{1}(t) \\
-\Lambda_{2}(t)+\beta_{2}(t) x_{5}(t) x_{1}(t)+\mu_{2}(t) x_{5}(t) \\
-\Lambda_{3}(t)+\beta_{3}(t) x_{6}(t) x_{2}(t)+\mu_{3}(t) x_{6}(t)
\end{array}\right) .
$$

We call the rearranged model system as "new model." According to Lemma 2, we easily know that the model system has a unique disease-free periodic solution $\tilde{x}^{*}(t)=$ $\left(S_{h}^{*}(t), 0, S_{s}^{*}(t), 0, S_{f}^{*}(t), 0\right)$. Then $x^{*}(t)=\left(0,0,0, S_{h}^{*}(t), S_{s}^{*}(t)\right.$, $\left.S_{f}^{*}(t)\right)$ is the unique disease-free periodic solution of the new model.

Let $C_{\omega}^{+}=\left\{\phi \in C\left(R, R_{+}^{3}\right) \mid \phi(t+\omega)=\phi(t)\right.$, for all $\left.t \in R\right\}$, which is equipped with maximum norm $\|\cdot\|_{\infty}$. It follows that the new infection matrix at $x^{*}(t)$ is

$$
\begin{aligned}
F(t) & =\left(\frac{\partial \mathscr{F}_{i}\left(t, x^{*}(t)\right)}{\partial x_{j}}\right)_{1 \leq i, j \leq 3} \\
& =\left(\begin{array}{ccc}
0 & 0 & \beta_{1}(t) S_{h}^{*}(t) \\
\beta_{2}(t) S_{s}^{*}(t) & 0 & 0 \\
0 & \beta_{3}(t) S_{f}^{*}(t) & 0
\end{array}\right),
\end{aligned}
$$

and the evolution of the initial infective members introduced at $x^{*}(t)$ is described by

$$
\begin{gathered}
\dot{y}(t)=-V(t) y, \quad t \neq t_{k}, k \in \mathbb{N}, \\
y\left(t_{k}^{+}\right)=P_{k} y\left(t_{k}\right), \quad t=t_{k}, \quad k \in \mathbb{N} .
\end{gathered}
$$

Let $Y(t, s)$ be the evolution operator of $(20)$ and define a linear operator $L$ on $C_{12}^{+}$by

$$
(L \phi)(t)=\int_{0}^{+\infty} Y(t, t-a) F(t-a) \phi(t-a) d a,
$$

$\forall t \in R$.

It follows from [15] that the basic reproductive number $R_{0}$ of the model system is given by $R_{0}=r(L)$. 
Following [15], by direct computation, we have

$$
\begin{aligned}
& P_{k}=\left(\begin{array}{ccc}
1 & 0 & 0 \\
0 & 1-\theta_{k} & 0 \\
0 & 0 & 1
\end{array}\right) \\
& Q_{k}=\left(\begin{array}{ccc}
1 & 0 & 0 \\
0 & 1-\theta_{k} & 0 \\
0 & 0 & 1
\end{array}\right), \\
& M(t)=\left(\frac{\partial f_{i}\left(t, x^{*}(t)\right)}{\partial x_{j}}\right)_{4 \leq i, j \leq 6} \\
& =\left(\begin{array}{ccc}
-\mu_{1}(t)-\alpha(t)-\delta(t) & 0 & 0 \\
0 & -\mu_{2}(t) & 0 \\
0 & 0 & -\mu_{3}(t)
\end{array}\right) \text {, }
\end{aligned}
$$

and

$$
\begin{aligned}
V(t) & =\left(\frac{\partial \mathscr{V}_{i}\left(t, x^{*}(t)\right)}{\partial x_{j}}\right)_{1 \leq i, j \leq 3} \\
& =\left(\begin{array}{ccc}
\mu_{1}(t)+\gamma(t) & 0 & 0 \\
0 & \mu_{2}(t) & 0 \\
0 & 0) & \mu_{3}(t)
\end{array}\right) .
\end{aligned}
$$

Obviously, $r\left(\Phi_{M Q_{k}}(12)\right)<1$ and $r\left(\Phi_{-V P_{k}}(12)\right)<1$, and then the assumptions $\left(H_{1}\right)-\left(H_{8}\right)$ in [15] hold. By Theorem 3.2 in [15], we can obtain that the following result.

Theorem 5. For the model system, the following statements are valid:

(i) $R_{0}=1 \Longleftrightarrow r\left(\Phi_{(F-V) P_{k}}(12)\right)=1$.

(ii) $R_{0}>1 \Longleftrightarrow r\left(\Phi_{(F-V) P_{k}}(12)\right)>1$.

(iii) $R_{0}<1 \Longleftrightarrow r\left(\Phi_{(F-V) P_{k}}(12)\right)<1$.

\section{Main Results}

In this section, we show that the basic reproductive number is a threshold parameter that determines dynamics of the disease. The first result shows that $C$. sinensis dies out if $R_{0}<$ 1 .

Theorem 6. The disease-free periodic solution $\tilde{x}^{*}(t)=$ $\left(S_{h}^{*}(t), 0, S_{s}^{*}(t), 0, S_{f}^{*}(t), 0\right)$ of the model system is asymptotically stable if $R_{0}<1$, and unstable if $R_{0}>1$.
Proof. The linearized system of the new model at the diseasefree periodic solution $x^{*}(t)$ is

$$
\begin{aligned}
& x^{\prime}(t)=\left(\begin{array}{cc}
F(t)-V(t) & 0 \\
A(t) & M(t)
\end{array}\right) x(t), \\
& x\left(t^{+}\right)=\left(\begin{array}{cc}
P_{k} & 0 \\
B_{k} & Q_{k}
\end{array}\right) x(t), \quad t=t_{k}, k \in \mathbb{N},
\end{aligned}
$$

where $F(t), V(t), M(t), P_{k}, Q_{k}$ are defined in (19), (22)-(24), and $A(t)$ and $B_{k}$ are zero matrices. Then the Floquet multipliers of system (25) are the eigenvalues of $r\left(\Phi_{(F-V) P_{k}}(12)\right)$ and $r\left(\Phi_{M Q_{k}}(12)\right)$. By Theorem 5, we have all Floquet multipliers of system (25) are less than 1 provided that $R_{0}<1$. Therefore, the disease-free periodic solution $\tilde{x}^{*}(t)$ of the model system is asymptotically stable. And if $R_{0}>1$ it is unstable. This completes the proof.

Theorem 7. If $R_{0}<1$, then the disease-free periodic solution $\tilde{x}^{*}(t)$ of the model system is globally asymptotically stable.

Proof. By Theorem 6, we know that if $R_{0}<1$, then $\tilde{x}^{*}(t)$ is locally asymptotically stable. Thus we only show that it attracts all nonnegative solution of the model system.

By Theorem 5, we know $R_{0}<1$ implies $r\left(\Phi_{(F-V) P_{k}}(12)\right)<$ 1. Thus there is a sufficiently small $\varepsilon>0$ such that

$$
r\left(\Phi_{\left(F-V+M_{\varepsilon}\right) P_{k}}(12)\right)<1
$$

where

$$
M_{\varepsilon}=\left(\begin{array}{ccc}
0 & 0 & \beta_{1}(t) \varepsilon \\
\beta_{2}(t) \varepsilon & 0 & 0 \\
0 & \beta_{3}(t) \varepsilon & 0
\end{array}\right) \text {. }
$$

Let $\left(S_{h}(t), I_{h}(t), S_{s}(t), I_{s}(t), S_{f}(t), I_{f}(t)\right)$ be any solution of the model system. In view of Lemma 1 , the first equation of system (3) yields

$$
\begin{aligned}
S_{h}^{\prime}(t) \leq & \left(\mu_{1}(t)+\delta(t)\right) N_{h} \\
& -\left(\alpha(t)+\mu_{1}(t)+\delta(t)\right) S_{h}(t) .
\end{aligned}
$$

Consider the following comparison system:

$$
\begin{aligned}
\widehat{S}_{h}^{\prime}(t)= & \left(\mu_{1}(t)+\delta(t)\right) N_{h} \\
& -\left(\alpha(t)+\mu_{1}(t)+\delta(t)\right) \widehat{S}_{h}(t), \\
\widehat{S}_{h}\left(0^{+}\right)= & S_{h}\left(0^{+}\right) .
\end{aligned}
$$

By [16], we know that the first equation of (29) admits a positive periodic solution $S_{h}^{*}(t)$ which is globally asymptotically stable. Thus $\widehat{S}_{h}(t) \longrightarrow S_{h}^{*}(t)$, as $t \longrightarrow+\infty$. By the comparison theorem, we have $S_{h}(t) \leq \widehat{S}_{h}(t)$. Hence, there exists a sufficiently large $\xi_{0}>0$ and given above $\varepsilon>0$, such that $S_{h}(t) \leq S_{h}^{*}(t)+\varepsilon$, for $t \geq \xi_{0}$. In the same way, we can also prove that $S_{f}(t) \leq S_{f}^{*}(t)+\varepsilon$, for $t \geq \xi_{0}^{\prime}$. 
From the model system, we have

$$
\begin{gathered}
S_{s}^{\prime}(t) \leq \Lambda_{2}(t)-\mu_{2}(t) S_{s}(t), \quad t \neq t_{k}, k \in Z, \\
S_{s}\left(t^{+}\right)=\left(1-\theta_{k}\right) S_{s}(t) \leq S_{s}(t), \quad t=t_{k}, k \in Z .
\end{gathered}
$$

By comparison theorem in impulsive equations and above method, we can obtain $S_{s}(t) \leq S_{s}^{*}(t)+\varepsilon$ for $t \geq \xi_{0}^{\prime \prime}$.

Let $\xi^{*}=\max \left\{\xi_{0}, \xi_{0}^{\prime}, \xi_{0}^{\prime \prime}\right\}$. According to above discussion, we have for $t \geq \xi^{*}$

$$
\begin{aligned}
& S_{h}(t) \leq S_{h}^{*}(t)+\varepsilon, \\
& S_{f}(t) \leq S_{f}^{*}(t)+\varepsilon, \\
& S_{s}(t) \leq S_{s}^{*}(t)+\varepsilon .
\end{aligned}
$$

From (3), (4), and (31), we have that for $t \geq \xi^{*}$,

$$
\begin{array}{ll}
I_{h}^{\prime}(t) & \leq \beta_{1}(t)\left(S_{h}^{*}+\varepsilon\right) I_{f}-\left(\mu_{1}(t)+\gamma(t)\right) I_{h}, \\
I_{s}^{\prime}(t) & \leq \beta_{2}(t)\left(S_{s}^{*}+\varepsilon\right) I_{h}-\mu_{2}(t) I_{s}, \\
I_{f}^{\prime}(t) \leq \beta_{3}(t)\left(S_{f}^{*}+\varepsilon\right) I_{s}-\mu_{3}(t) I_{f}, & \\
I_{h}\left(t_{k}^{+}\right) & t \neq t_{k}, k \in \mathbb{N}, \\
I_{s}\left(t_{k}^{+}\right) & =\left(1-t_{k}\right), \\
I_{f}\left(t_{k}^{+}\right) & =I_{f}\left(t_{k}\right),
\end{array}
$$

$$
t=t_{k}, k \in \mathbb{N} .
$$

Consider the following linear approximation system:

$$
\begin{aligned}
& \widehat{x}^{\prime}(t)=\left(F(t)-V(t)+M_{\varepsilon}\right) \hat{x}(t), \quad t \neq t_{k}, k \in \mathbb{N}, \\
& \widehat{x}\left(t_{k}^{+}\right)=P_{k} \widehat{x}\left(t_{k}\right), \quad t=t_{k}, k \in \mathbb{N},
\end{aligned}
$$

where $F, V, P_{k}$ are defined as (19), (23), and (24).

By Lemma 4, we have that there exists a positive, 12period function $\widehat{x}^{*}(t)$ such that $\widehat{x}(t)=e^{\eta t} \widehat{x}^{*}(t)$ is a solution of system (33), where $\eta=(1 / 12) \ln r\left(\Phi_{\left(F-V+M_{\varepsilon}\right) P_{k}}(12)\right), \widehat{x}(t)=$ $\left(\widehat{I}_{h}(t), \widehat{I}_{s}(t), \widehat{I}_{f}(t)\right)$. It follows from $(26)$ that $\eta<0$. Therefore, we have $\widehat{x}(t) \longrightarrow 0$ as $t \longrightarrow+\infty$. This implies that the zero solution of system (33) is globally attractive if $R_{0}<1$.

For any nonnegative initial value $\left(I_{h}\left(0^{+}\right), I_{s}\left(0^{+}\right), I_{f}\left(0^{+}\right)\right)^{T}$ of system (32), there exists a sufficiently large $a>0$ such that $\left(I_{h}\left(0^{+}\right), I_{s}\left(0^{+}\right), I_{f}\left(0^{+}\right)\right)^{T} \leq a \hat{x}\left(0^{+}\right)$holds. Applying the comparison principle, we have $\left(I_{h}(t), I_{s}(t), I_{f}(t)\right)^{T} \leq a \hat{x}(t)$, for all $t>\xi_{0}$, where $a \hat{x}(t)$ is also the solution of system (33). Therefore, we have $I_{h}(t) \longrightarrow 0, I_{s}(t) \longrightarrow 0$ and $I_{f}(t) \longrightarrow 0$, as $t \longrightarrow+\infty$. By the theory of asymptotically semiflows, it follows that

$$
\begin{aligned}
& \lim _{t \rightarrow+\infty} S_{h}(t)=S_{h}^{*}(t), \\
& \lim _{t \longrightarrow+\infty} S_{s}(t)=S_{s}^{*}(t)
\end{aligned}
$$

Therefore the disease-free periodic solution $x^{*}(t)$ of the model system is globally asymptotically stable. This completes the proof.

The subsequent result shows that the disease is uniformly persistent if $R_{0}>1$.

Define

$$
\begin{aligned}
X & :=\left\{\left(S_{h}, I_{h}, S_{s}, I_{s}, S_{f}, I_{f}\right) \in R^{6} \mid S_{i} \geq 0, I_{i} \geq 0, i\right. \\
& =h, s, f\} \\
X_{0} & :=\left\{\left(S_{h}, I_{h}, S_{s}, I_{s}, S_{f}, I_{f}\right) \in X \mid I_{h}>0, I_{s}>0, I_{f}\right. \\
& >0\}, \quad \partial X_{0}:=X \backslash X_{0} .
\end{aligned}
$$

Let $P: X \longrightarrow X$ be the Poincaré map associated with the model system, that is,

$$
P\left(x^{0}\right)=u\left(12^{+}, x^{0}\right), \quad \forall x^{0} \in X,
$$

where $x^{0}=\left(S_{h}^{0}, I_{h}^{0}, S_{s}^{0}, I_{s}^{0}, S_{f}^{0}, I_{f}^{0}\right)$ and $u\left(t, x^{0}\right)$ is the unique solution of the model system with $u\left(0^{+}, x^{0}\right)=x^{0}$. It is easy to see that

$$
P^{m}\left(x^{0}\right)=u\left(12 m^{+}, x^{0}\right), \quad \forall m \in N .
$$

Letting $U^{*}=\left(S_{h}^{*}\left(0^{+}\right), 0, S_{s}^{*}\left(0^{+}\right), 0, S_{f}^{*}\left(0^{+}\right), 0\right)$, we have

$$
P^{m}\left(U^{*}\right)=u\left(12 m^{+}, U^{*}\right), \quad \forall m \in N .
$$

According to Lemma 1 , we can easily see that $X$ and $X_{0}$ are positively invariant, and Poincaré map $P$ is point dissipative.

Next, we establish the following lemma which will be useful in subsequent main result.

Lemma 8. If the basic reproductive number $R_{0}>1$, then there exists $\varepsilon^{*}>0$, such that for any $x^{0} \in X_{0}$ with $\left\|x^{0}-U^{*}\right\| \leq \varepsilon^{*}$, we have

$$
\limsup _{m \rightarrow \infty} d\left(P^{m}\left(x^{0}\right), U^{*}\right) \geq \varepsilon^{*}
$$

Proof. In view of Theorem 5, we know that $R_{0}>1$ if and only if $r\left(\Phi_{(F-V) P_{k}}(12)\right)>1$, and then there is a $\varepsilon>0$ sufficiently small such that

$$
r\left(\Phi_{\left(F-V-M_{\varepsilon}\right) P_{k}}(12)\right)>1,
$$

where $M_{\varepsilon}$ is given in (27).

By the continuity of the solutions with respect to the initial values, there exists $\varepsilon^{*}>0$, such that for any $x^{0} \in X_{0}$ with $\left\|x^{0}-U^{*}\right\| \leq \varepsilon^{*}$ and $t \in[0,12)$,

$$
\left\|u\left(t, x^{0}\right)-u\left(t, U^{*}\right)\right\| \leq \varepsilon .
$$

Next, we claim that (39) holds. Suppose the claim is not valid. Then there exists $x^{0} \in X_{0}$ such that

$$
\limsup _{m \rightarrow \infty} d\left(P^{m}\left(x^{0}\right), U^{*}\right)<\varepsilon^{*} .
$$


Without loss of generality, we assume that

$$
d\left(P^{m}\left(x^{0}\right), U^{*}\right)<\varepsilon^{*}, \quad \text { for all } m \geq 0 .
$$

It follows from (41) and (43) that

$$
\left\|u\left(t, P^{m}\left(x^{0}\right)\right)-u\left(t, U^{*}\right)\right\|<\varepsilon,
$$

$\forall m \geq 0, \forall t \in[0,12)$.

For any $t \geq 0$, there exist nonnegative integer $m^{\prime}$ and $T_{1} \epsilon$ $[0,12)$ such that $t=12 m^{\prime}+T_{1}$. From (44), we have

$$
\begin{aligned}
& \left\|u\left(t, x^{0}\right)-u\left(t, U^{*}\right)\right\| \\
& \quad=\left\|u\left(T_{1}, P^{m \prime}\left(x^{0}\right)\right)-u\left(T_{1}, U^{*}\right)\right\|<\varepsilon .
\end{aligned}
$$

Note that $\left(S_{h}(t), I_{h}(t), S_{s}(t), I_{s}(t), S_{f}(t), I_{f}(t)\right)=u\left(t, x^{0}\right)$. Thus

$$
\begin{aligned}
\left\|I_{h}(t)\right\| & <\varepsilon, \\
\left\|I_{s}(t)\right\| & <\varepsilon, \\
\left\|I_{f}(t)\right\| & <\varepsilon
\end{aligned}
$$

for all $t \geq 0$.

By the first equation of (3) and (46), we have

$$
\begin{aligned}
S_{h}^{\prime}(t) \geq & \left(\mu_{1}(t)+\delta(t)\right) N_{h}-\beta_{1}(t) S_{h}(t) \varepsilon \\
& -\left(\alpha(t)+\mu_{1}(t)+\delta(t)\right) S_{h}(t)-\delta(t) \varepsilon .
\end{aligned}
$$

By comparison theorem, we have $S_{h}(t) \geq \widetilde{S}_{h}(t)$ and $\widetilde{S}_{h}(t) \longrightarrow$ $S_{h}^{*}(t)$ as $\varepsilon \longrightarrow 0$, where $\widetilde{S}_{h}(t)$ is the solution of the following system:

$$
\begin{aligned}
\widetilde{S}_{h}^{\prime}(t)= & \left(\mu_{1}(t)+\delta(t)\right) N_{h}-\beta_{1}(t) S_{h}(t) \varepsilon-\delta(t) \varepsilon \\
& -\left(\alpha(t)+\mu_{1}(t)+\delta(t)\right) S_{h}(t), \\
\widetilde{S}_{h}\left(0^{+}\right)= & S_{h}\left(0^{+}\right) .
\end{aligned}
$$

Therefore, for above mentioned $\varepsilon$, there exists $T_{2}>0$ sufficiently large such that

$$
S_{h}(t) \geq \widetilde{S}_{h}(t) \geq S_{h}^{*}(t)-\varepsilon, \quad \text { for } t \geq T_{2} .
$$

Using the same method we can get there exists $T_{3} \geq T_{2}$ sufficiently large such that

$$
\begin{aligned}
& S_{f}(t) \geq \widetilde{S}_{f}(t) \geq S_{f}^{*}(t)-\varepsilon, \\
& S_{s}(t) \geq \widetilde{S}_{s}(t) \geq S_{s}^{*}(t)-\varepsilon
\end{aligned}
$$

From (3), (4), (49), and (50), we obtain for $t \geq T_{3}$,

$$
\begin{aligned}
& I_{h}\left(t_{k}^{+}\right)=I_{h}\left(t_{k}\right), \\
& I_{s}\left(t_{k}^{+}\right)=\left(1-\theta_{k}\right) I_{s}\left(t_{k}\right), \\
& I_{f}\left(t_{k}^{+}\right)=I_{f}\left(t_{k}\right),
\end{aligned}
$$$$
I_{h}^{\prime}(t) \geq \beta_{1}(t)\left(S_{h}^{*}(t)-\varepsilon\right) I_{f}(t)
$$$$
-\left(\mu_{1}(t)+\gamma(t)\right) I_{h}(t)
$$$$
I_{s}^{\prime}(t) \geq \beta_{2}(t)\left(S_{s}^{*}(t)-\varepsilon\right) I_{h}(t)-\mu_{2}(t) I_{s}(t),
$$$$
I_{f}^{\prime}(t) \geq \beta_{3}(t)\left(S_{f}^{*}(t)-\varepsilon\right) I_{s}(t)-\mu_{3}(t) I_{f}(t),
$$$$
t \neq t_{k}, k \in \mathbb{N}
$$$$
t=t_{k}, k \in \mathbb{N} \text {. }
$$

Consider the following impulsive linear approximation system:

$$
\begin{aligned}
& \tilde{x}^{\prime}(t)=\left(F(t)-V(t)-M_{\varepsilon}\right) \tilde{x}(t), \quad t \neq t_{k}, k \in \mathbb{N}, \\
& \tilde{x}\left(t_{k}^{+}\right)=P_{k} \tilde{x}\left(t_{k}\right), \quad t=t_{k}, k \in \mathbb{N} .
\end{aligned}
$$

By Lemma 4, we know that there exists a positive, 12-period function $\tilde{x}^{*}(t)=\left(\widetilde{I}_{h}^{*}(t), \widetilde{I}_{s}^{*}(t), \widetilde{I}_{f}^{*}(t)\right)$ such that $\tilde{x}(t)=e^{\eta_{1} t} \tilde{x}^{*}(t)$ is a solution of (52), where $\tilde{x}(t)=\left(\widetilde{I}_{h}(t), \widetilde{I}_{s}(t), \widetilde{I}_{f}(t)\right)$ and $\eta_{1}=$ $(1 / 12) \ln r\left(\Phi_{\left(F-V-M_{\varepsilon}\right) P_{k}}(12)\right)$. It follows from (40) that $\eta_{1}>0$. Obviously, we can choose $12 \widetilde{k}>T_{3}$ and a proper $b>0$ such that

$$
\begin{aligned}
\widetilde{I}_{h}\left(12 \widetilde{k}^{+}\right) & \geq b \widetilde{I}_{h}^{*}\left(0^{+}\right), \\
\widetilde{I}_{s}\left(12 \widetilde{k}^{+}\right) & \geq b \widetilde{I}_{s}^{*}\left(0^{+}\right), \\
\text {and } \widetilde{I}_{f}\left(\left(12 \widetilde{k}^{+}\right)\right. & \geq b \widetilde{I}_{f}^{*}\left(0^{+}\right) .
\end{aligned}
$$

By the comparison theorem we have that for all $t \geq 12 \widetilde{k}$,

$$
\begin{gathered}
I_{h}(t) \geq \widetilde{I}_{h}(t) \geq b \mathrm{e}^{\eta_{1}(t-12 \tilde{k})} \widetilde{I}_{h}^{*}(t-12 \widetilde{k}), \\
I_{s}(t) \geq \widetilde{I}_{s}(t) \geq b e^{\eta_{1}(t-12 \widetilde{k})} \widetilde{I}_{s}^{*}(t-12 \widetilde{k}), \\
I_{f}(t) \geq \widetilde{I}_{f}(t) \geq b e^{\eta_{1}(t-12 \tilde{k})} \widetilde{I}_{f}^{*}(t-12 \widetilde{k}) .
\end{gathered}
$$

Then, we can obtain that $I_{h}(t) \longrightarrow+\infty, I_{s}(t) \longrightarrow+\infty$, and $I_{f}(t) \longrightarrow+\infty$, as $t \longrightarrow+\infty$ which is a contradiction. The proof of Lemma 8 is completed. 
Theorem 9. If the basic reproductive number $R_{0}>1$, then there exist constants $\delta_{i}>0(i=1,2,3)$ such that for any solution of the model system with initial value

$$
\left(S_{h}^{0}, I_{h}^{0}, S_{s}^{0}, I_{s}^{0}, S_{f}^{0}, I_{f}^{0}\right) \in X_{0}
$$

satisfies

$$
\begin{aligned}
& \liminf _{t \rightarrow+\infty} I_{h}(t) \geq \delta_{1}, \\
& \liminf _{t \longrightarrow+\infty} I_{s}(t) \geq \delta_{2},
\end{aligned}
$$

and $\liminf _{t \rightarrow+\infty} I_{f}(t) \geq \delta_{3}$.

Proof. Denote

$$
\begin{aligned}
M_{\partial} & =\left\{\left(S_{h}^{0}, I_{h}^{0}, S_{s}^{0}, I_{s}^{0}, S_{f}^{0}, I_{f}^{0}\right)\right. \\
& \left.\in \partial X_{0} \mid H^{m}\left(S_{h}^{0}, I_{h}^{0}, S_{s}^{0}, I_{s}^{0}, S_{f}^{0}, I_{f}^{0}\right) \in \partial X_{0}, m \in \mathbb{N}\right\} .
\end{aligned}
$$

We claim that

$$
\begin{aligned}
M_{\partial} & =\left\{\left(S_{h}, 0, S_{s}, 0, S_{f}, 0\right) \in \partial X_{0} \mid S_{h} \geq 0, S_{s} \geq 0, S_{f}\right. \\
& \geq 0\} .
\end{aligned}
$$

Obviously, $M_{\partial} \supseteq\left\{\left(S_{h}, 0, S_{s}, 0, S_{f}, 0\right) \in \partial X_{0} \mid S_{h} \geq\right.$ $\left.0, S_{s} \geq 0, S_{f} \geq 0\right\}$. Thus we only need to prove $M_{\partial} \backslash$ $\left\{\left(S_{h}, 0, S_{s}, 0, S_{f}, 0\right) \in \partial \mathrm{X}_{0} \mid S_{h} \geq 0, S_{s} \geq 0, S_{f} \geq 0\right\}=\emptyset$. If it is not true, then there exists a point $x^{0}=\left(S_{h}^{0}, I_{h}^{0}, S_{s}^{0}, I_{s}^{0}, S_{f}^{0}, I_{f}^{0}\right) \in$ $M_{\partial} \backslash\left\{\left(S_{h}, 0, S_{s}, 0, S_{f}, 0\right) \in \partial X_{0} \mid S_{h} \geq 0, S_{s} \geq 0, S_{f} \geq 0\right\}$.

There are six cases to consider: (i) $I_{h}>0, I_{s}=0, I_{f}=0$, (ii) $I_{h}=0, I_{s}>0, I_{f}=0$, (iii) $I_{h}=0, I_{s}=0, I_{f}>0$, (iv) $I_{h}=0, I_{s}>0, I_{f}>0$, (v) $I_{h}>0, I_{s}=0, I_{f}>0$, (vi) $I_{h}>0$, $I_{s}>0, I_{f}=0$.

Case A. For case (i), that is, $I_{h}>0, I_{s}=0, I_{f}=0$, it is easily seen that $I_{h}(t)>0$ and $S_{s}(t)>0, S_{f}(t)>0$ for all $t>0$. Then from the fourth equation of $(3),\left.\left(d I_{s}(t) / d t\right)\right|_{t=0}=$ $\beta_{2}(0) S_{s}\left(0^{+}\right) I_{h}\left(0^{+}\right)>0$. Thus $I_{s}(t)>0$ for $0<t \ll 1$. It follows from the sixth equation of (3) that $I_{f}(t)>0$ for $0<t \ll 1$. It is easy to obtain that $\left(S_{h}(t), I_{h}(t), S_{s}(t), I_{s}(t), S_{f}(t), I_{f}(t)\right) \notin$ $\partial X_{0}$ for $0<t \ll 1$. This is a contradiction. Using the same method, we can prove the second and the third cases.

Case B. For case (iv), that is, $I_{h}=0, I_{s}>0, I_{f}>0$, it is easily seen that $I_{s}(t)>0, I_{f}(t)>0$, and $S_{h}(t)>0$ for all $t>0$. Then from the second equation of system (3), $\left.\left(d I_{h}(t) / d t\right)\right|_{t=0}=\beta_{1}(0) S_{h}\left(0^{+}\right) I_{f}\left(0^{+}\right)>0$. Obviously, $\left(S_{h}(t), I_{h}(t), S_{s}(t), I_{s}(t), S_{f}(t), I_{f}(t)\right) \notin \partial X_{0}$ for $0<t \ll 1$. This is a contradiction. We can also discuss the last two cases using the same method.

From the above discussion, we have that, for any initial $x^{0} \notin\left\{\left(S_{h}^{0}, 0, S_{s}^{0}, 0, S_{f}^{0}, 0\right) \mid S_{h} \geq 0, S_{s} \geq 0, S_{f} \geq 0\right\}$, then $x^{0} \notin$ $M_{\partial}$. Thus $M_{\partial}=\left\{\left(S_{h}, 0, S_{s}, 0, S_{f}, 0\right) \in \partial X_{0} \mid S_{h} \geq 0, S_{s} \geq\right.$ $\left.0, S_{f} \geq 0\right\}$.

Obviously, Piocaré map $P$ has a global attractor $U^{*}$, $U^{*}$ is an isolated invariant set in $X$ and $W^{s}\left(U^{*}\right) \cap X_{0}=$ $\varnothing$. Further, $U^{*}$ is acyclic in $M_{\partial}$ and every solution in $M_{\partial}$ converges to $U^{*}$. By Lemma 8 , we know that $P$ is weakly uniformly persistent with respect to $\left(X_{0}, \partial X_{0}\right)$. According to Zhao [17], we have that $P$ is uniformly persistent with respect to $\left(X_{0}, \partial X_{0}\right)$. That is, there exist constants $\delta_{i}>0(i=1,2,3)$ such that for any solution of the model system with initial value

$$
\left(S_{h}^{0}, I_{h}^{0}, S_{s}^{0}, I_{s}^{0}, S_{f}^{0}, I_{f}^{0}\right) \in X_{0}
$$

satisfies

$$
\begin{array}{r}
\liminf _{t \rightarrow+\infty} I_{h}(t) \geq \delta_{1}, \\
\liminf _{t \longrightarrow+\infty} I_{s}(t) \geq \delta_{2}, \\
\text { and } \liminf _{t \rightarrow+\infty} I_{f}(t) \geq \delta_{3} .
\end{array}
$$

The proof of Theorem 9 is completed.

\section{Numerical Simulations}

In this section, we will make numerical simulations in Matlab. Using the theory of impulsive equations and analysis method, dynamic behavior of the model has been studied and a threshold for a disease to be extinct or endemic has been established.

Before carrying out the numerical simulations, we have to estimate the model parameters. Some data are taken from literature $[14,18]$. Some data are estimated from the clonorchiasis surveillance data of Foshan city, Guangdong province, China. The values of the model parameters are listed in Table 1.

Set $q=2, t_{1}=4, t_{2}=6$. In view of the clonorchiasis surveillance data, the initial value of the model system is taken as follows: $S_{h}(0)=2.368 \times 10^{6}, I_{h}(0)=1.6038 \times 10^{6}$, $S_{s}(0)=7.488 \times 10^{10}, I_{s}(0)=1.872 \times 10^{10}, S_{f}(0)=1.76 \times 10^{8}$, $I_{f}(0)=4.4 \times 10^{7}$.

If we fix $\theta_{1}=0$ and $\theta_{2}=0$, that is, no snail control strategy is implemented. With numerical simulation, we get $R_{0}=1.8858>1$. If we fix $\theta_{1}=0.2$ and $\theta_{2}=0$, that is, $20 \%$ snail-killing once a year is carried out, which results in $R_{0}=1.0719>1$, these, of course, lead to the persistence of the disease, as clearly indicated by Figures 3 and 4 . If we fix $\theta_{1}=0.2$ and $\theta_{2}=0.1$, then $R_{0}=0.9140<1$. This suggests the extinction of the disease (see Figure 5).

Figure 6 shows the sensitivity of the basic reproductive number $R_{0}$ to the elimination rate of snails $\theta_{1}$ and $\theta_{2}$. It is apparent that the elimination rate of snails are very sensitive to $R_{0}$ when $\theta_{i}<0.2(i=1,2)$.

Health education, snail control, and feces treatment are three effective strategies to control clonorchiasis. Parameters $\alpha$ (or $\delta), \theta_{i}$, and $\beta_{2}$ reflect the efforts of health education, snail control, and feces treatment, respectively. In Table 2, six projects are studied to determine the impact of control strategies on the basic reproductive number. The first and second projects are only considered the health education strategy. If $\alpha$ is doubled and $\delta$ is half, then $R_{0}$ decreases from 
TABLE 1: The values of parameters in the model system.

\begin{tabular}{|c|c|c|c|}
\hline Parameter & Value & Unit & Reference \\
\hline$\mu_{1}(t)$ & 0.0011 & month $^{-1}$ & {$[18]$} \\
\hline$\beta_{1}(t)$ & $7.5 \times 10^{-11}+2 \times 10^{-11} \sin \frac{\pi t}{6}$ & month $h^{-1}$ & Estimation \\
\hline$\alpha(t)$ & $0.0167+0.002 \sin \frac{\pi t}{6}$ & month $^{-1}$ & Estimation \\
\hline$\delta(t)$ & $0.02778+0.009 \sin \frac{\pi t}{6}$ & month $^{-1}$ & Estimation \\
\hline$\gamma(t)$ & $0.076+0.006 \sin \frac{\pi t}{6}$ & month $h^{-1}$ & [14] \\
\hline$\Lambda_{2}(t)$ & $1.3 \times 10^{9}+1.0 \times 10^{8} \sin \frac{\pi t}{6}$ & month $^{-1}$ & {$[10,14]$} \\
\hline$\mu_{2}(t)$ & $0.01389+0.01 \sin \frac{\pi t}{6}$ & month $h^{-1}$ & [19] \\
\hline$\beta_{2}(t)$ & $3.8 \times 10^{-8}+5 \times 10^{-10} \sin \frac{\pi t}{6}$ & month $^{-1}$ & Estimation \\
\hline$\Lambda_{3}(t)$ & $4.6 \times 10^{6}+2 \times 10^{5} \sin \frac{\pi t}{6}$ & month $^{-1}$ & Estimation \\
\hline$\beta_{3}(t)$ & $1.78 \times 10^{-12}+5 \times 10^{-14} \sin \frac{\pi t}{6}$ & month ${ }^{-1}$ & Estimation \\
\hline$\mu_{3}(t)$ & $0.0208+0.101 \sin \frac{\pi t}{6}$ & month $h^{-1}$ & {$[10]$} \\
\hline
\end{tabular}
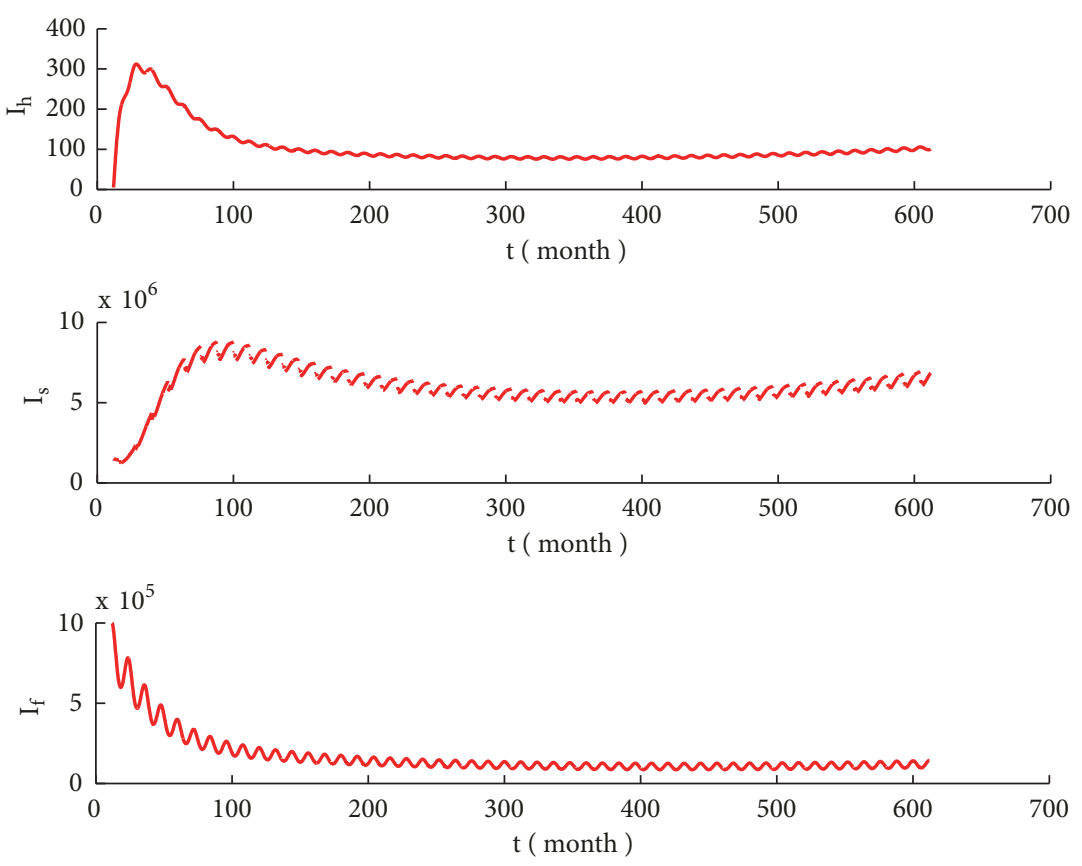

Figure 3: This figure shows movement paths of $I_{h}, I_{s}$, and $I_{f}$ as functions of time $t \cdot R_{0}=1.8858>1$, where parameters $\theta_{1}=0$ and $\theta_{2}=0$. The disease is permanent.

1.8858 to 1.4855 . And if $\alpha$ is tripled and $\delta$ is reduced by onethird, then $R_{0}$ decreases from 1.8858 to 1.2192 . Combining health education and snail control strategies in the third project, $\alpha$ is doubled and $\delta$ is in half. $\theta_{1}=0.14$, by computer simulation. It is shown that $R_{0}$ decreases to 0.9674 . This indicates that the disease will die out. Combining snail control and faeces treatment strategies, the last project shows $R_{0}$ also becomes less than 1 . From Table 2, it is evident that health educations, snail control and faeces treatment are helpful for the disease control. They can all decrease the fraction of infected individuals; however, snail-killing is the most effective way to control the transmission of clonorchiasis.

\section{Conclusions and Discussion}

Clonorchiasis has been one of significant public health threats in China. Facing up to the epidemic situation, both the central and local governments have been exploring an effective and sustainable strategy on the control of clonorchiasis in endemic areas. Various prevention and control measures have been proposed by many researchers. Chemotherapy with praziquantel is one of vital strategies, but the use of praziquantel for humans and animals has been only temporarily effective. There is no doubt that the chemotherapy-based control strategy would no longer be an ideal measure. 
TABLE 2: Comparison of different control strategies.

\begin{tabular}{|c|c|c|c|c|c|c|}
\hline Plan & $\begin{array}{l}\text { Health education } \\
\text { efforts }\end{array}$ & $\begin{array}{c}\text { Snail } \\
\text { control } \theta_{1}\end{array}$ & $\begin{array}{c}\text { Faeces } \\
\text { treatment }\end{array}$ & $R_{0}$ & Extinction & Permanence \\
\hline 1 & $\begin{array}{l}\alpha(t) \times 2 \\
\delta(t) \div 2\end{array}$ & 0 & $\beta_{2}(t) \times 1$ & 1.4855 & No & Yes \\
\hline 2 & $\begin{array}{l}\alpha(t) \times 3 \\
\delta(t) \div 3\end{array}$ & 0 & $\beta_{2}(t) \times 1$ & 1.2192 & No & Yes \\
\hline 3 & $\begin{array}{l}\alpha(t) \times 2 \\
\delta(t) \div 2\end{array}$ & 0.14 & $\beta_{2}(t) \times 1$ & 0.9674 & Yes & No \\
\hline 4 & $\begin{array}{l}\alpha(t) \times 1 \\
\delta(t) \div 1\end{array}$ & 0 & $\beta_{2}(t) \div 2$ & 1.4968 & No & Yes \\
\hline 5 & $\begin{array}{l}\alpha(t) \times 2 \\
\delta(t) \div 2\end{array}$ & 0 & $\beta_{2}(t) \div 2$ & 1.1790 & No & Yes \\
\hline 6 & $\begin{array}{l}\alpha(t) \times 1 \\
\delta(t) \div 1\end{array}$ & 0.14 & $\beta_{2}(t) \div 2$ & 0.9747 & Yes & No \\
\hline
\end{tabular}
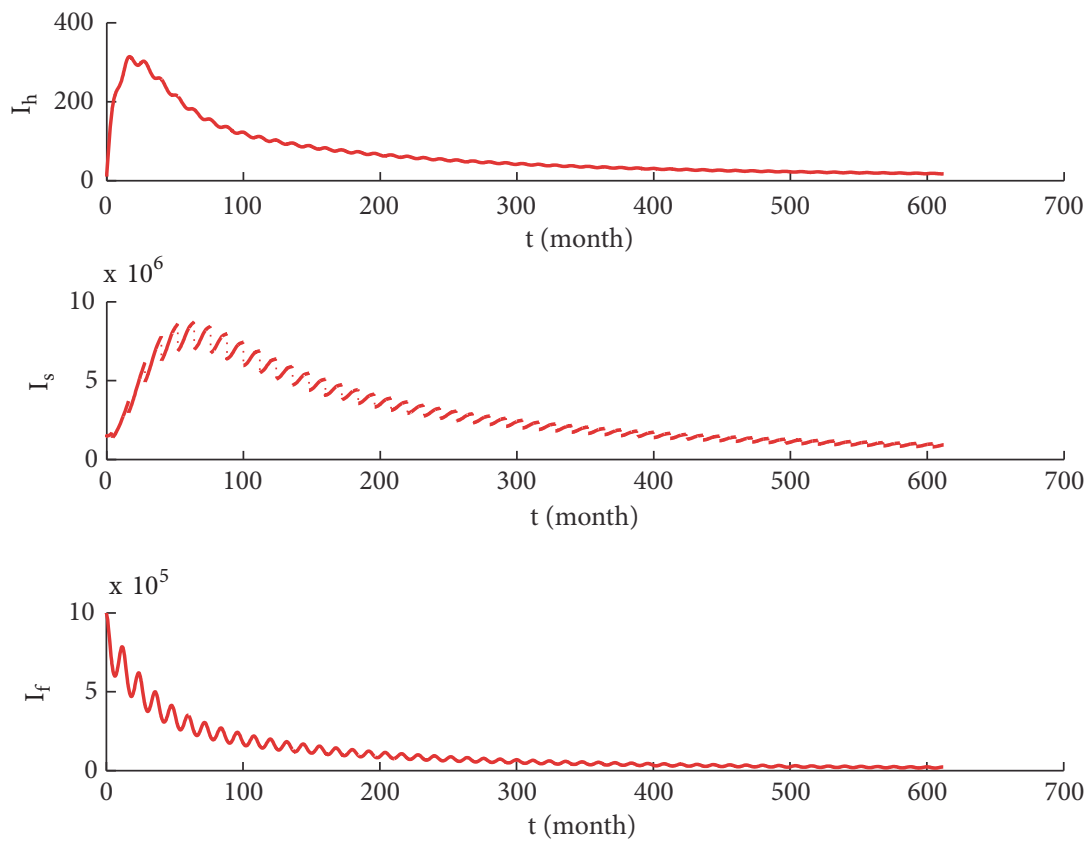

FIgURE 4: This figures show movement paths of $I_{h}, I_{s}$, and $I_{f}$ as functions of time $t . R_{0}=1.0719>1$, where parameters $\theta_{1}=0.2$ and $\theta_{2}=0$. The disease is permanent.

Currently, the integrated control strategy mainly focuses on ensuring fish cooked well prior to consumption, health education, and improvement of sanitation to prevent parasite eggs reaching fish habitats. Controlling snail is widely used to control of schistosomiasis [20], but it has not yet been applied to control clonorchiasis in practice, even if it has been theoretically confirmed. In this paper, we proposed a dynamic clonorchiasis model with impulsive snail-killing to study the effects of snail control strategy on the transmission of clonorchiasis. We also derived the basic reproductive number for general epidemic model with seasonality and impulsive control, which extended previous works on such models in that the impulsive control actions can be carried out at several time points within a period. Furthermore, we performed the numerical simulation and sensitivity analysis to recognize the impact of crucial model parameters on $R_{0}$. We found that controlling snail was the best effective method for eliminating clonorchiasis.

As Hepatitis B virus mainly attacks the liver, C. sinensis infection also causes liver diseases, as well as biliary conditions. Both Hepatitis B and C. sinensis are present in almost the same regions in China [8]. Therefore, using HBV/CI coinfection model to assess different tools and strategies for large-scale control of Hepatitis B and clonorchiasis should be an interesting issue which will be considered in the future. 

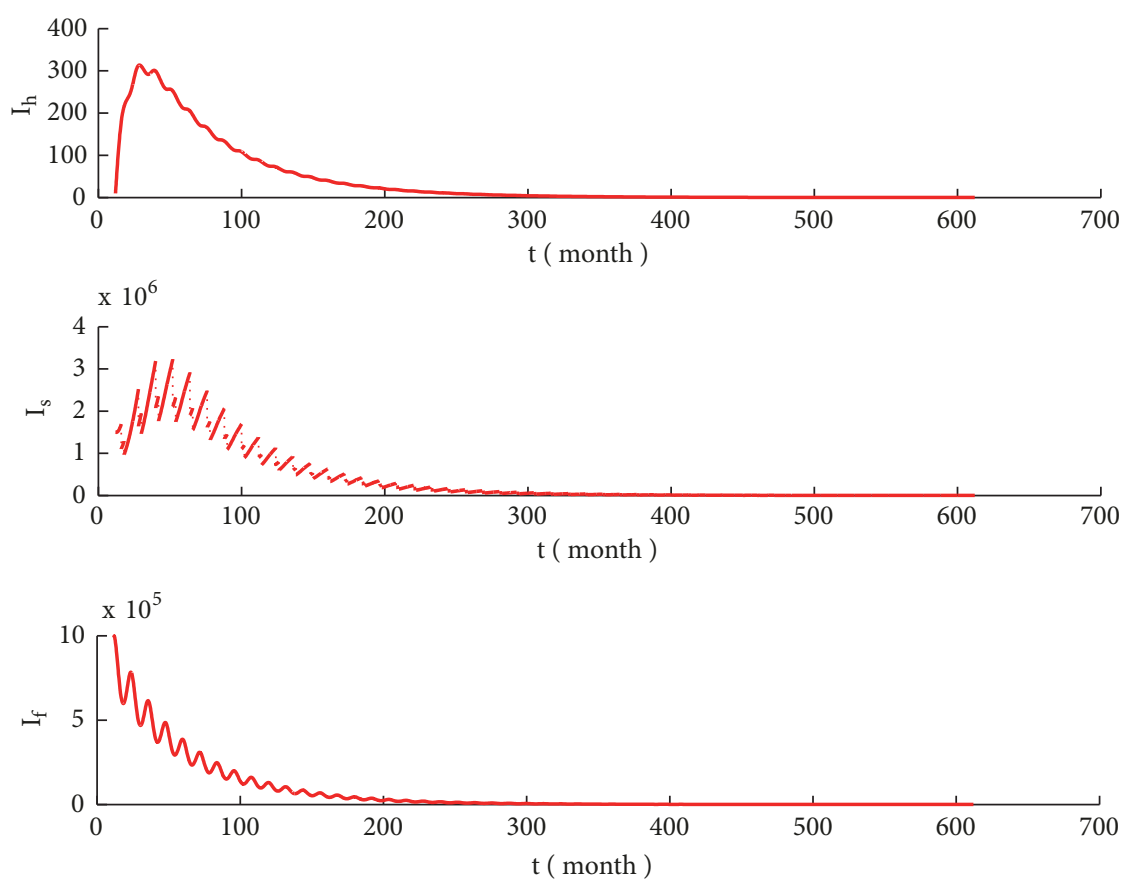

Figure 5: This figure shows movement paths of $I_{h}, I_{s}$, and $I_{f}$ as functions of time $t . R_{0}=0.9140<1$, where parameters $\theta_{1}=0.2$ and $\theta_{2}=0.1$. The disease will be distinct.

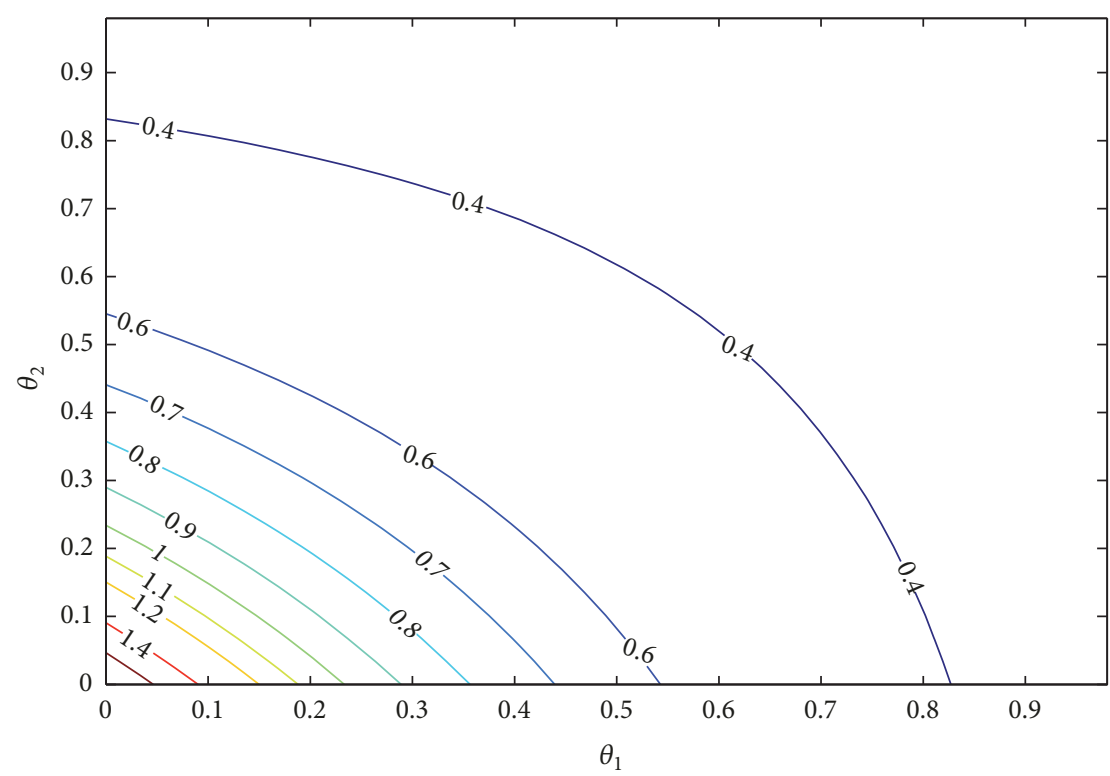

FIGURE 6: The graph is a contour plot showing the regions in the $\left(\theta_{1}, \theta_{2}\right)$ plane in which $R_{0}$ falls in different intervals.

\section{Appendix}

Proof of Lemma 2. Integrating and solving the first equation of system (11) between pulses for $n \omega+t_{i}<t \leq n \omega+t_{i+1}(i=$ $0,1, \ldots, q-1)$

$$
\begin{aligned}
z(t)= & z\left(n \omega+t_{i}^{+}\right) e^{-\int_{n \omega+t_{i}}^{t} b(\tau) d \tau} \\
& +e^{-\int_{n \omega+t_{i}}^{t} b(\tau) d \tau} \int_{n \omega+t_{i}}^{t} a(s) e^{\int_{n \omega+t_{i}}^{s} b(u) d u} d s,
\end{aligned}
$$

where $z\left(n \omega+t_{i}^{+}\right)=\left(1-\theta_{i}\right) z\left(n \omega+t_{i}\right)$. It follows from the above equation and the second equation of system (11) that

$$
\begin{gathered}
z\left(n \omega+t_{1}^{+}\right)=\left(1-\theta_{1}\right)\left(z\left(n \omega+t_{0}\right) e^{-\int_{n \omega+t_{0}}^{n \omega+t_{1}} b(\tau) d \tau}\right. \\
\left.+e^{-\int_{n \omega+t_{0}}^{n \omega+t_{1}} b(\tau) d \tau} \int_{n \omega+t_{0}}^{n \omega+t_{1}} a(s) e^{\int_{n \omega+t_{0}}^{s} b(u) d u} d s\right),
\end{gathered}
$$


and

$$
\begin{aligned}
& z\left(n \omega+t_{2}^{+}\right)=\left(1-\theta_{2}\right)\left(z\left(n \omega+t_{1}^{+}\right) e^{-\int_{n \omega+t_{1}}^{n \omega+t_{2}} b(\tau) d \tau}\right. \\
& \left.+e^{-\int_{n \omega+t_{1}}^{n \omega+t_{2}} b(\tau) d \tau} \int_{n \omega+t_{1}}^{n \omega+t_{2}} a(s) e^{\int_{n \omega+t_{1}}^{s} b(u) d u} d s\right)=(1 \\
& \left.-\theta_{1}\right)\left(1-\theta_{2}\right) z\left(n \omega+t_{0}\right) e^{-\int_{n \omega+t_{0}}^{n \omega+t_{2}} b(\tau) d \tau}+\left(1-\theta_{1}\right) \\
& \cdot\left(1-\theta_{2}\right) e^{-\int_{n \omega+t_{1}}^{n \omega+t_{2}} b(\tau) d \tau} \int_{n \omega+t_{0}}^{n \omega+t_{1}} a(s) e^{\int_{n \omega+t_{0}}^{s} b(u) d u} d s \\
& +\left(1-\theta_{2}\right) e^{-\int_{n \omega+t_{1}}^{n \omega+t_{2}} b(\tau) d \tau} \int_{n \omega+t_{1}}^{n \omega+t_{2}} a(s) e^{\int_{n \omega+t_{1}}^{s} b(u) d u} d s .
\end{aligned}
$$

Using the inductive method, we know $z\left(n \omega+t_{q}^{+}\right)=z((n+$ 1) $\left.\omega+t_{0}^{+}\right)$and

$$
\begin{aligned}
z\left(n \omega+t_{q}^{+}\right)= & \prod_{l=1}^{q}\left(1-\theta_{l}\right) z\left(n \omega+t_{0}\right) e^{-\int_{n \omega+t_{0}}^{n \omega+t_{q}} b(\tau) d \tau} \\
& +\sum_{l=1}^{q} \prod_{j=l}^{q}\left(1-\theta_{j}\right) e^{-\int_{n \omega+t_{j-1}}^{n \omega+t_{q}} b(\tau) d \tau} \\
& \cdot \int_{n \omega+t_{j-1}}^{n \omega+t_{j}} a(s) e^{\int_{n \omega+t_{j-1}}^{s} b(u) d u} d s .
\end{aligned}
$$

Set $U_{n}=z\left(n \omega+t_{0}\right)$. From (A.4) and $t_{q}-t_{0}=\omega$, we have

$$
\begin{aligned}
& U_{n+1}=\prod_{l=1}^{q}\left(1-\theta_{l}\right) U_{n} e^{-\int_{n \omega+t_{0}}^{n \omega+t_{q}} b(\tau) d \tau} \\
& +\sum_{l=1}^{q} \prod_{j=l}^{q}\left(1-\theta_{j}\right) e^{-\int_{n \omega+t_{j-1}}^{n \omega+t_{q}} b(\tau) d \tau} \\
& \cdot \int_{n \omega+t_{j-1}}^{n \omega+t_{j}} a(s) e^{\int_{n \omega+t_{j-1}}^{s} b(u) d u} d s \triangleq f\left(U_{n}\right),
\end{aligned}
$$

where $f$ is the stroboscopic map. It is easy to see that system (A.5) has a unique positive equilibrium

$$
\begin{aligned}
z_{0}^{*} & =z\left(0^{+}\right)=\left[1-\prod_{l=1}^{q}\left(1-\theta_{l}\right) e^{\int_{n \omega+t_{0}}^{n \omega+t_{q}}-b(\tau) d \tau}\right]^{-1} \\
& \times \sum_{l=1}^{q} \prod_{j=l}^{q}\left(1-\theta_{j}\right) e^{\int_{n \omega+t_{j-1}}^{n \omega+t_{q}}-b(\tau) d \tau} \\
& \cdot \int_{n \omega+t_{j-1}}^{n \omega+t_{j}} a(s) e^{\int_{n \omega+t_{j-1}}^{s} b(\tau) d \tau} d s .
\end{aligned}
$$

Since $f\left(U_{n}\right)$ is a straight line with slope less than 1 , we obtain that $z_{0}^{*}$ is globally asymptotically stable. It implies that the corresponding periodic solution of system (11) $z^{*}(t)$ is globally asymptotically stable. The proof of Lemma 2 is completed.

\section{Data Availability}

The data used to support the findings of this study are available from the corresponding author upon request.

\section{Conflicts of Interest}

The authors declare that there are no conflicts of interest regarding the publication of this paper.

\section{Acknowledgments}

The work by Shujing Gao was partially supported by The Natural Science Foundation of China (11561004).

\section{References}

[1] R. Anderson and R. May, Infectious Diseases of Humans: Dynamics and Control, Oxford University Press, USA, 1991.

[2] S. Hong and Y. Fang, "Clonorchis sinensis and clonorchiasis, an update," Parasitology International, vol. 61, no. 1, pp. 17-24, 2012.

[3] N. Bacaër and R. Ouifki, "Growth rate and basic reproduction number for population models with a simple periodic factor," Mathematical Biosciences, vol. 210, no. 2, pp. 647-658, 2007.

[4] M. Qian, Y. Chen, S. Liang, G. Yang, and X. Zhou, "The global epidemiology of clonorchiasis and its relation with cholangiocarcinoma," Infectious Diseases of Poverty, vol. 1, no. 4, pp. 1-11, 2012.

[5] Z. Lun, R. B. Gasser, D. Lai et al., "Clonorchiasis: a key foodborne zoonosis in China," The Lancet Infectious Diseases, vol. 5, no. 1, pp. 31-41, 2005.

[6] J. Keiser and J. Utzinger, "Food-borne trematodiases," Clinical Microbiology Reviews, vol. 22, no. 3, pp. 466-483, 2009.

[7] B. Sripa, S. Kaewkes, P. M. Intapan, W. Maleewong, and P. J. Brindley, "Food-borne trematodiases in Southeast Asia epidemiology, pathology, clinical manifestation and control," Advances in Parasitology, vol. 72, pp. 305-350, 2010.

[8] M. Qian, Y. Chen, and F. Yan, "Time to tackle clonorchiasis in China," Infectious Diseases of Poverty, vol. 2, no. 4, pp. 1-4, 2013.

[9] P. Wei, Studies on the killing effect of Clonorchis sinensis metacercariae by non-heated treatment and metacercariae histochemical properties analysis, MSc thesis, Guangxi University, Nanning, China, 2013.

[10] Q. Gaun, Z. Huang, X. Huang et al., "The infection status and epidemiological characteristics of clonorchiasis in Foshan 19802010," South China Journal of Preventive Medicine, vol. 41, no. 3, pp. 276-279, 2015.

[11] B. X. Yang, Z. Z. Chen, L. C. Huo et al., "Investigation on epidemiology of clonorchiasis and discussion of control strategy and measures," Chinese Journal of Public Health, vol. 10, no. 3, pp. 303-306, 1994.

[12] R. Yuan, J. Huang, X. Zhang, and S. Ruan, "Modeling the transmission dynamics of clonorchiasis in Foshan, China," Scientific Reports, vol. 8, article 15176, pp. 1-9, 2018.

[13] Y. Dai and S. Gao, "Threshold and stability results for clonorchiasis epidemic model," Journal of Science, Technology and Environment, vol. 2, no. 2, pp. 1-13, 2013.

[14] T. Li, Z. Yang, and M. Wang, "Correlation between clonorchiasis incidences and climatic factors in Guangzhou, China," Parasites \& Vectors, vol. 7, no. 1, article 29, 2014. 
[15] S. Gao, Y. Tu, and J. Wang, "Basic reproductive number for a general hybrid epidemic model," Advances in Difference Equations, vol. 2018, article 310, pp. 1-9, 2018.

[16] A. d'Onofrio, "On pulse vaccination strategy in the SIR epidemic model with vertical transmission," Applied Mathematics Letters, vol. 18, no. 7, pp. 729-732, 2005.

[17] X. Q. Zhao, Dynamical Systems in Population Biology, Springer, New York, NY, USA, 2003.

[18] E. T. Chiyaka and W. Garira, "Mathematical analysis of the transmission dynamics of schistosomiasis in the human-snail hosts," Journal of Biological Systems, vol. 17, no. 3, pp. 397-423, 2009.

[19] S. Gao, Y. He, Y. Liu, G. Yang, and X. Zhou, "Field transmission intensity of Schistosoma japonicum measured by basic reproduction ratio from modified Barbours model," Parasites Vectors, vol. 6, article 141, 2013.

[20] T. Lin, Q. W. Jiang, D. D. Lin et al., "Classification study on the marshland in endemic areas of Schistosoma japonicum using satellite TM image data," Chinese Journal of Preventive Medicine, vol. 35, no. 5, pp. 312-314, 2001. 


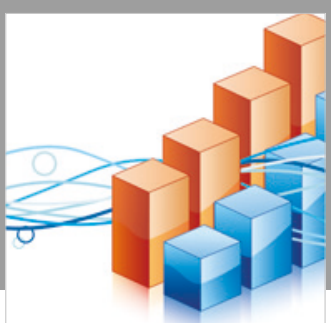

Advances in

Operations Research

\section{-n-m}
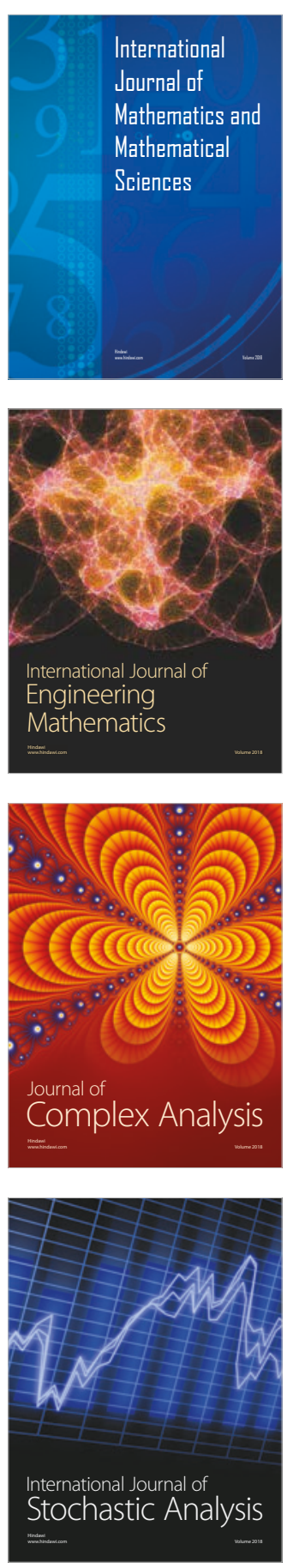
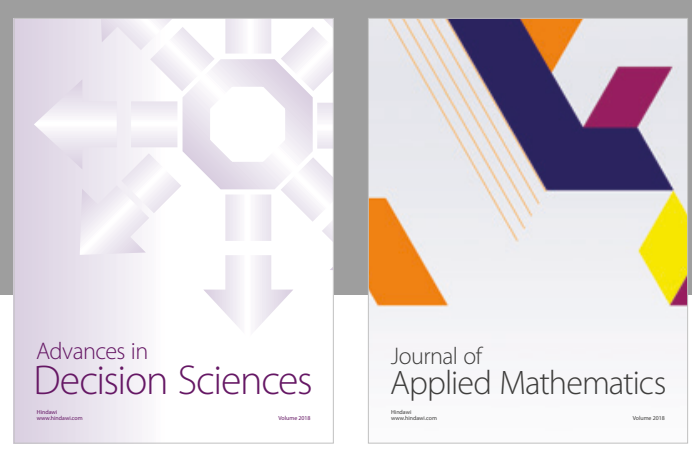

Journal of

Applied Mathematics
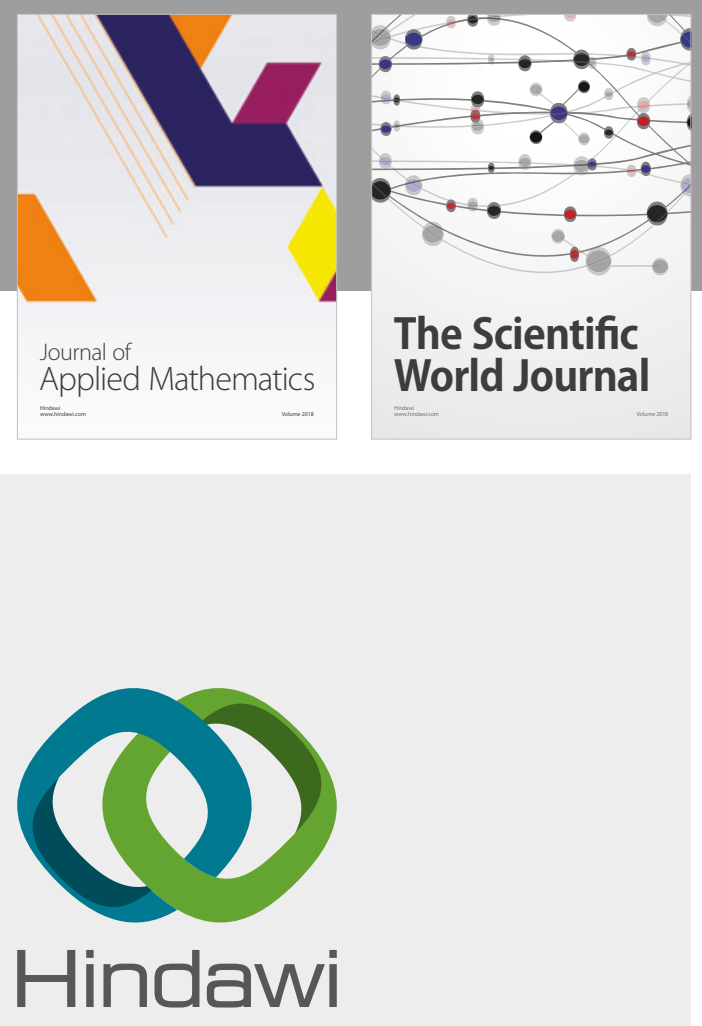

Submit your manuscripts at

www.hindawi.com

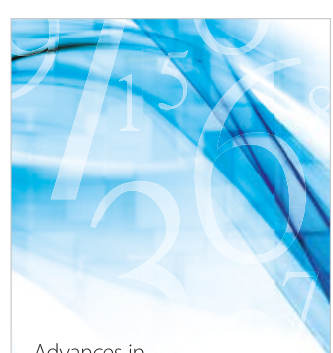

Advances in
Numerical Analysis
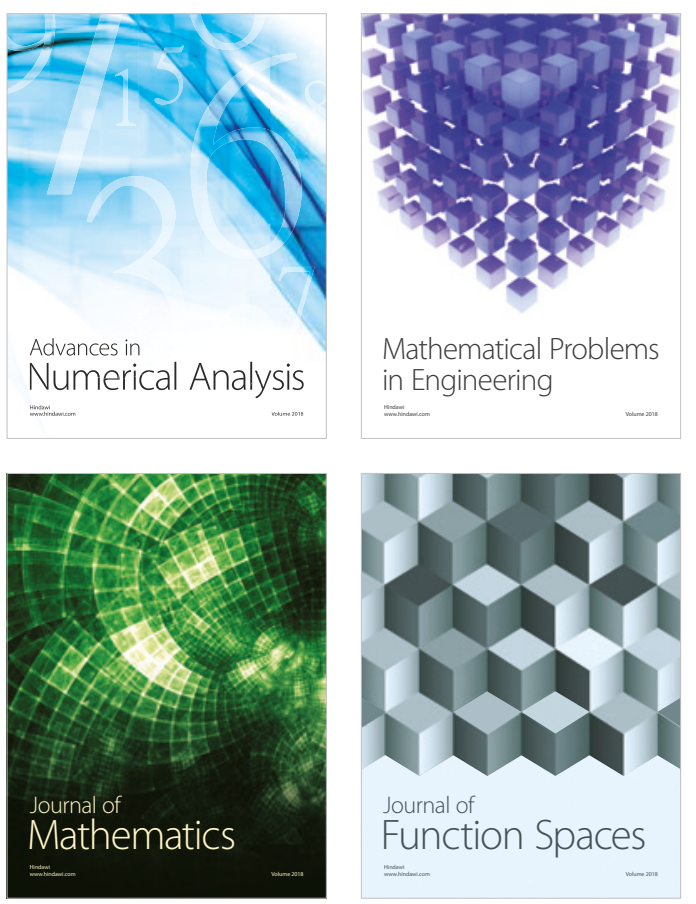

Mathematical Problems in Engineering

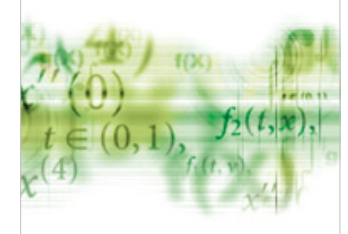

International Journal of

Differential Equations

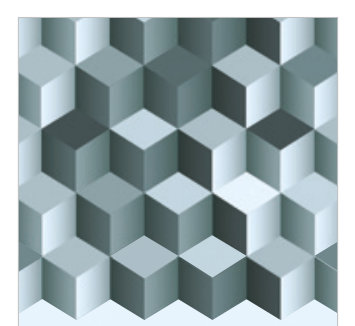

Journal of

Function Spaces
The Scientific

World Journal

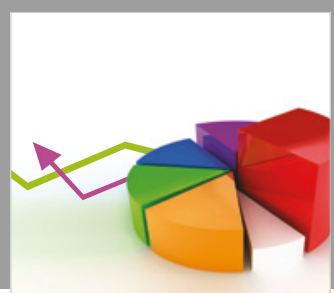

Journal of

Probability and Statistics
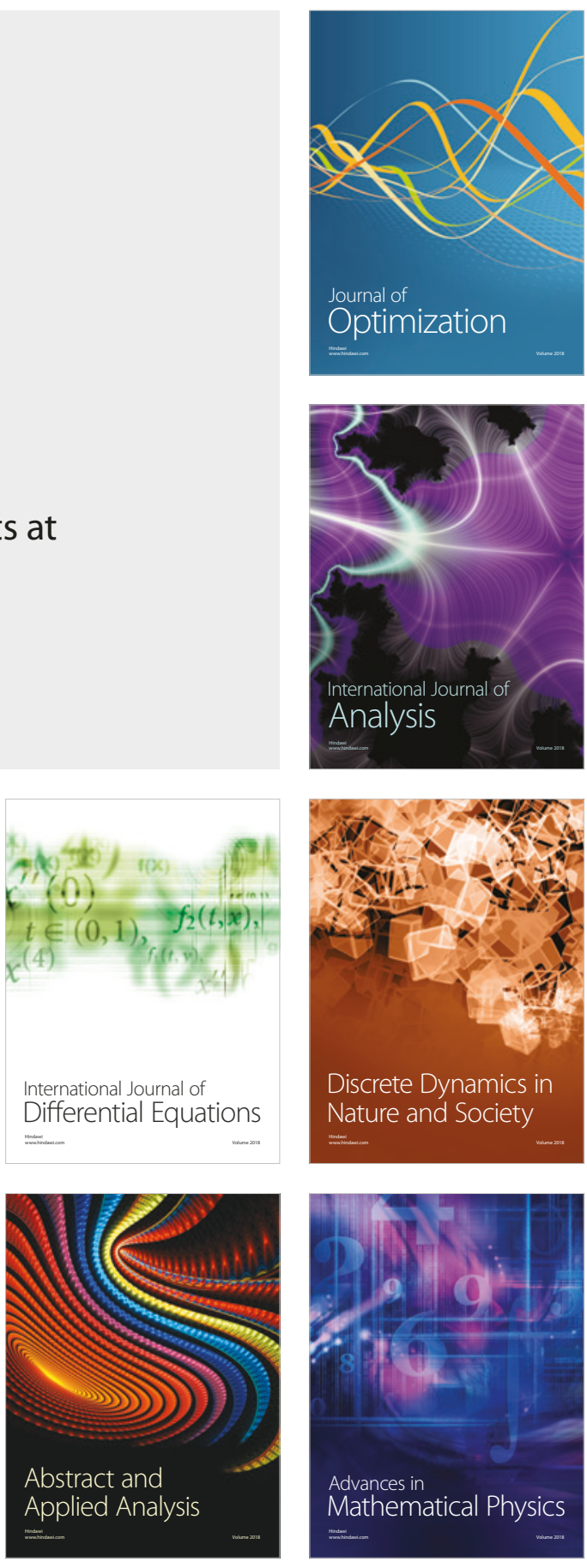